\title{
Simultaneous Listeria monocytogenes septicaemia in mother and pre-term infant
}

\author{
P. BURGESS \\ M.B.Ch.B., D.C.H. \\ C. S. SMITH \\ M.B.Ch.B., M.R.C.P.
}

\author{
A. Ben-Musa \\ M.B.Ch.B. \\ F. HARRIS \\ M.D., M.Med(Paed.), F.R.C.P.E.
}

Mill Road Maternity Hospital, Mill Road, Liverpool L6 2AH

\begin{abstract}
Summary
Although infection by Listeria monocytogenes during pregnancy with subsequent infection of the fetus is well known, it is uncommon to encounter acute maternal septicaemia with the onset of premature labour and delivery of an infected infant. Such a case is described of maternal septicaemia with an infected liveborn preterm infant who was successfully treated and recovered.
\end{abstract}

\section{Case report}

A 22-year-old Caucasian primigravida in a hitherto normal 28-week pregnancy developed intermittent fever with generalized headache, facial paraesthesia, photophobia and 2 episodes of vomiting. Her parents suffered from migraine and the patient ascribed her headaches to the same cause. Her general practitioner prescribed paracetamol. She attended routine ante-natal clinic at 30 weeks and was admitted as her symptoms were unchanged. Her temperature was $35 \cdot 3^{\circ} \mathrm{C}$, BP $100 / 60$ $\mathrm{mmHg}$ and uterus $30-32$ weeks by size. The fetal heart sounds were normal and the urine showed no abnormality. Further examination was unremarkable.

On the following day her temperature was $38.2^{\circ} \mathrm{C}$. Full blood count, throat swabs, urine analysis and culture, and blood culture were performed. $\mathrm{Hb}$, $10.2 \mathrm{~g} / \mathrm{dl} ; \mathrm{PCV} 34 \%$; white cell count $11 \times 10^{9} / 1$ with $70 \%$ neutrophils, $26 \%$ lymphocytes, $2 \%$ monocytes and $2 \%$ eosinophils.

Later that day her temperature rose to $39.4^{\circ} \mathrm{C}$ and ampicillin was started. However, that evening she commenced premature labour at 31 weeks' gestation and was given a single i.m. dose of dexamethasone, $12 \mathrm{mg}$. A live female infant (2.31 $\mathrm{kg}$ ) was delivered with meconium-stained liquor after a labour of $3 \mathrm{hr} 40 \mathrm{~min}$.

The infant gasped at birth but became apnoeic at $2 \mathrm{~min}$. She was intubated and ventilated with oxygen for $7 \mathrm{~min}$. No meconium was seen in the pharynx.
The Apgar score was 9 at $10 \mathrm{~min}$. Because of the maternal pyrexia, swabs and blood were taken for culture from the infant and a lumbar puncture was performed. The spinal fluid was uniformly bloodstained and no organisms were seen or cultured. At $12 \mathrm{hr}$ the infant was tachypnoeic and began to grunt intermittently. There was minimal costal recession and she remained pink in air. The heart rate was $160 /$ min with no evidence of cardiac failure. The liver was palpable $2 \mathrm{~cm}$ below the costal margin and the spleen $1 \mathrm{~cm}$. Chest $X$-ray showed inflammatory changes in the right lower zone. The baby was treated with intravenous fluids, penicillin and gentamicin. By $36 \mathrm{hr}$ the grunting had ceased and at the age of 2 days the chest X-ray was clear. Three days after delivery Listeria monocytogenes was identified from maternal and infant blood cultures and also from the infant's axillary, throat and umbilical swabs. As the organism was demonstrated to be only moderately sensitive to penicillin, ampicillin was substituted and continued for 7 days. The infant's subsequent course was uneventful.

\section{Discussion}

Human listeriosis is an uncommon infection. Between 1970 and 1976 the mean number of cases reported (Public Health Laboratory Service (PHLS) unpublished) annually in Great Britain was 30, ranging from 20 to 40 .

Listeriosis is also an uncommon cause of premature labour. Hood (1961) reported 2 cases in 66 mothers with acute or threatened abortion screened by swabs from the uterus, cervix and vagina and in 21 of their infants screened by throat swab, blood culture and CSF examination. The 2 cases identified were a mother and her infant.

In 1972 the PHLS reported 25 cases where $L$. monocytogenes was cultured. These included a mother whose blood culture grew $L$. monocytogenes and her infant delivered at 30 weeks' gestation. The 
infant died from meningitis and septicaemia and $L$. monocytogenes was recovered from his CSF, upper respiratory tract and umbilicus.

In spite of being infrequently encountered, the condition remains important because of the poor prognosis for the infant unless a very early diagnosis is made and treatment commenced. Ray and Wedgewood (1964) reviewed the literature of neonatal listeriosis and described 6 new cases. In the 69 cases reviewed there was a mortality of $54 \%$. Delta (1965) summarized 15 further cases which were not included in the earlier review. Five of these cases had died and information on outcome was not available for 3 of the remainder.

An improved prognosis was reported by Ahlfors et al. (1977) who described 5 cases, 4 of whom survived. All were associated with maternal fever during labour.

In 1977 the PHLS recorded 47 cases of human listeriosis in Britain. Eleven of these were neonates, and 4 were premature infants, stillborn or dying soun after birth. Listeria was found in the maternal genital tract of 2 of these cases and no instance of simultaneous maternal septicaemia was reported.

Listeriosis thus carries a significant mortality for newborns and for the fetus in utero. Therefore, fever and malaise in a pregnant woman, especially if premature labour begins, warrants a blood culture to exclude a systemic listerial infection. The bacteriology laboratory should be warned if the infection is suspected as the culture may be mistaken by the unwary for $\beta$-haemolytic Streptococcus or nonpathogenic diphtheroids. Ampicillin is the recommended treatment for listerial infection in the newborn period. However, unless there is bacteriological confirmation of the diagnosis at the commencement of treatment, ampicillin should be given together with gentamicin or kanamycin pending positive identification of the organism.

\section{Acknowledgment}

We thank Mr R. D. Atlay, M.R.C.O.G. for permission to report the details of the mother.

\section{References}

Ahlfors, C.E., Goetzman, B.W., Halstead, C.C., Sherman, M.P. \& WENNBERG, R.P. (1977) Neonatal listeriosis. American Journal of Diseases of Children, 131, 405.

DeLTA, B.G. (1965) Neonatal listeriosis and the problem of retrieving medical information. Pediatrics, 35, 358.

HOOD, M. (1961) Listeriosis as an infection of pregnancy manifested in the newborn. Pediatrics, 27, 390.

RAY, G.C. \& WEDGWOOD, R.J. (1964) Neonatal listeriosis. Pediatrics, 34, 378. 